\title{
Results from the First Science Run of the ZEPLIN-III Dark Matter Search Experiment
}

\author{
V. N. Lebedenko* ${ }^{1}$ H. M. Araújo, ${ }^{1,2}$ E. J. Barnes,${ }^{3}$ A. Bewick, ${ }^{1}$ R. Cashmore,${ }^{4}$ V. Chepel,${ }^{5}$ A. Currie,${ }^{1}$ D. Davidge,${ }^{1}$ \\ J. Dawson, ${ }^{1}$ T. Durkin, ${ }^{2}$ B. Edwards, ${ }^{1,2}$ C. Ghag, ${ }^{3}$ M. Horn,${ }^{1}$ A. S. Howard, ${ }^{1}$ A. J. Hughes,${ }^{2}$ W. G. Jones,${ }^{1}$ \\ M. Joshi, ${ }^{1}$ G. E. Kalmus, ${ }^{2}$ A. G. Kovalenko, ${ }^{6}$ A. Lindote, ${ }^{5}$ I. Liubarsky,${ }^{1}$ M. I. Lopes,${ }^{5}$ R. Lüscher, ${ }^{2}$ P. Majewski, ${ }^{2}$ \\ A. StJ. Murphy ${ }^{3}$ F. Neves $,{ }^{5}, 1$ J. Pinto da Cunha, ${ }^{5}$ R. Preece, ${ }^{2}$ J. J. Quenby,${ }^{1}$ P. R. Scovell,${ }^{3}$ C. Silva,${ }^{5}$ \\ V. N. Solovov, ${ }^{5}$ N. J. T. Smith, ${ }^{2}$ P. F. Smith, ${ }^{2}$ V. N. Stekhanov,${ }^{6}$ T. J. Sumner ${ }^{\dagger},{ }^{1}$ C. Thorne, ${ }^{1}$ and R. J. Walker ${ }^{1}$ \\ ${ }^{1}$ Blackett Laboratory, Imperial College London, UK \\ ${ }^{2}$ Particle Physics Department, Rutherford Appleton Laboratory, Chilton, UK \\ ${ }^{3}$ School of Physics and Astronomy, SUPA, University of Edinburgh, UK \\ ${ }^{4}$ Brasenose College, University of Oxford, UK \\ ${ }^{5}$ LIP-Coimbra \&3 Department of Physics of the University of Coimbra, Portugal \\ ${ }^{6}$ Institute for Theoretical and Experimental Physics, Moscow, Russia
}

(Dated: November 1, 2018)

\begin{abstract}
The ZEPLIN-III experiment in the Palmer Underground Laboratory at Boulby uses a $12 \mathrm{~kg}$ twophase xenon time projection chamber to search for the weakly interacting massive particles (WIMPs) that may account for the dark matter of our Galaxy. The detector measures both scintillation and ionisation produced by radiation interacting in the liquid to differentiate between the nuclear recoils expected from WIMPs and the electron recoil background signals down to $\sim 10 \mathrm{keV}$ nuclear recoil energy. An analysis of $847 \mathrm{~kg}$.days of data acquired between February $27^{\text {th }} 2008$ and May $20^{\text {th }} 2008$ has excluded a WIMP-nucleon elastic scattering spin-independent cross-section above $8.1 \times 10^{-8} \mathrm{pb}$ at $60 \mathrm{GeVc}^{-2}$ with a $90 \%$ confidence limit. It has also demonstrated that the two-phase xenon technique is capable of better discrimination between electron and nuclear recoils at low-energy than previously achieved by other xenon-based experiments.
\end{abstract}

\section{INTRODUCTION}

\section{A. Motivation}

Searches for weakly interacting massive particles (WIMPs) are motivated by the coming together of unification schemes, such as supersymmetry, which predict new particle species, and extensive observational evidence which demonstrates the need for additional nonbaryonic gravitational mass within the Universe. That the WIMPs of supersymmetry naturally fulfill this need is remarkably persuasive. Indeed, WIMPs occur in other frameworks too. As a generic class of particle they are assumed to only interact non-gravitationally with baryonic matter via the weak interaction. Whilst this offers a mechanism for energy transfer and hence detection, it also implies rather low event rates and energy deposits: $<0.1$ events/day $/ \mathrm{kg}$ and $<50 \mathrm{keV}$ respectively. This dictates the use of sensitive underground experiments capable of specifically identifying energy deposits due to elastic scattering of incoming particles from target nuclei. ZEPLIN-III is the latest in a progressive series of instruments designed to push steadily the sensitivity limits by exploring alternative approaches using xenon-based targets [1, 2].

\footnotetext{
*Deceased

${ }^{\dagger}$ Corresponding author; address: High Energy Physics Group, Blackett Laboratory, Imperial College London, SW7 2BW, UK. Email: t.sumner@imperial.ac.uk
}

\section{B. ZEPLIN-III}

ZEPLIN-III is a two-phase (liquid/gas) xenon timeprojection chamber specifically designed to search for dark matter WIMPs. Its design and performance details have already been presented elsewhere [3, 4] and only a brief reminder is given here. The experiment is operating $1100 \mathrm{~m}$ underground. The active volume is a disc of $35 \mathrm{~mm}$ thickness and $\sim 190 \mathrm{~mm}$ diameter which contains $\sim 12 \mathrm{~kg}$ of liquid xenon above an array of 31 2-inch diameter photomultipliers (PMTs). The PMTs employed during this first science run were ETL D730/9829Q [5], and they were used to record both the rapid scintillation signal, S1, and a delayed second signal, S2, produced by proportional electroluminescence in the gas phase above the liquid [6]. The PMT array was immersed in the liquid viewing upwards. The electric field in the target volume was defined by a cathode wire grid $36 \mathrm{~mm}$ below the liquid surface and an anode plate $4 \mathrm{~mm}$ above the surface in the gas phase. These two electrodes alone produce the drift field in the liquid $(3.9 \mathrm{kV} / \mathrm{cm})$, the field for extraction of the charge from the surface, and the electroluminescence field in the gas $(7.8 \mathrm{kV} / \mathrm{cm})$. A fiducial volume for WIMP searches was defined by using a time window for delays between S1 and S2, which selected a depth slice within the liquid, and by $2-\mathrm{D}$ position reconstruction from the PMT signals to select a radial boundary at $150 \mathrm{~mm}$. The time window was set between $500 \mathrm{~ns}$ and 13,000 ns which selected depths between $1.29 \mathrm{~mm}$ and $33.43 \mathrm{~mm}$. These together defined a fiducial volume containing $6.5 \mathrm{~kg}$ of xenon. 
The PMT signals were digitised at $2 \mathrm{~ns}$ sampling over a time segment of $\pm 18 \mu \mathrm{s}$ either side of the trigger point. Each PMT signal was fed into two 8-bit digitisers (ACQIRIS DC265) with a $\times 10$ gain difference between them provided by fast amplifiers (Phillips Scientific 770), to obtain both high and low sensitivity read-out covering a wide dynamic range. The PMT array was operated from a common HV supply with attenuators (Phillips Scientific 804) used to normalise their individual gains. The trigger was created from the shaped sum signal of all the PMTs. For nuclear recoil interactions the trigger was always caused by an S2 signal for energies up to $\mathrm{S} 1=40 \mathrm{keVee}$, where keVee is an energy unit referenced to the equivalent S1 signal produced by $122 \mathrm{keV}$ $\gamma$-rays from ${ }^{57} \mathrm{Co}$. The trigger threshold was $\sim 11$ ionisation electrons and this corresponded to $\sim 0.2 \mathrm{keV}$ for electron recoils (for nuclear recoils see Section IIID 2). This $\mathrm{S} 2$ threshold was set to avoid excessive triggers from single electron emission events and from electron and nuclear recoils whose primaries would otherwise have been undetectable as they fall below the S1 detection threshold.

The xenon target was contained within a vessel itself located within a vacuum jacket both made from lowbackground oxygen-free copper. Cooling was provided by a 40 litre liquid nitrogen reservoir, also made from copper, inside the vacuum jacket. Thermal stability to $<0.5^{\circ} \mathrm{C}$ was achieved over the entire run by controlling the flow of cold nitrogen boil-off gas through the baseflange of the xenon vessel. Pressure stability to $2 \%$ was maintained. The ZEPLIN-III detector was completely surrounded by a shield of $30 \mathrm{~cm}$ thick polypropylene and $20 \mathrm{~cm}$ thick lead, giving $10^{5}$ attenuation factors for both $\gamma$-rays and neutrons from the cavern walls. Dedicated access through the shield was provided for the radioactive calibration source delivery, instrument levelling screws and pipe-work to the external gas purification system.

\section{Science Data}

WIMP-search data were collected over 83 days of continuous operation in the Boulby Laboratory starting on $27^{\text {th }}$ February 2008. An $84 \%$ live time was achieved during the science run and some $847 \mathrm{~kg}$.days of raw data were collected from the $12 \mathrm{~kg}$ target volume. ${ }^{57} \mathrm{Co}$ calibration measurements were made every day. Nuclear recoil calibrations were made with an AmBe neutron source at the beginning and end of the 83 day period ( $5 \mathrm{hrs}$ each). A typical event, from a neutron elastic scattering interaction in the liquid with $\mathrm{S} 1=5 \mathrm{keVee}$, is shown in Figure 1 as recorded through the highsensitivity sum channel. A short Compton calibration was performed using a ${ }^{137} \mathrm{Cs}$ source at the beginning of the run with a much longer run at the end (122 hrs). Ten percent of the science data (every $10^{\text {th }}$ file) were used to develop initial data analysis and selection cuts, to establish the level of the electron-recoil background,

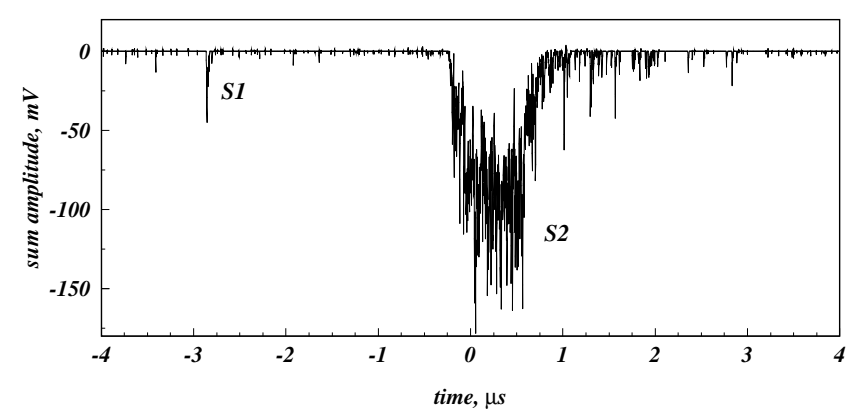

FIG. 1: Segment of the high-sensitivity summed waveform for a neutron elastic scattering event with $\mathrm{S} 1=5 \mathrm{keVee}$, showing a small primary pulse (S1) preceding a large secondary pulse (S2). Some PMT after-pulsing and, possibly, single electron emission can be seen following S2. Note that only excursions $>3 \mathrm{rms}$ on individual channels are added into the summed waveform. See later text for more detailed discussion of some of these points.

and to define the boundaries for the WIMP-search box and its acceptance. At first, the remaining $90 \%$ of the science data were retained unopened to carry out a 'blind' analysis, but these data were eventually used for perfecting some data-selection cuts as detailed below, making the final analysis non-blind.

Pulse-finding algorithms were used to identify signals in the 62 waveforms (independently for each PMT and for high and low sensitivity channels). These were then categorised as S1 or S2 candidates based on a pulse width parameter (charge mean arrival time, $\tau$ ): scintillation pulses are much shorter $(\tau \lesssim 40 \mathrm{~ns})$ than electroluminescence pulses, with durations corresponding to the drift time across the gas gap ( $\tau \sim 550 \mathrm{~ns})$. Viable S1 and S2 candidates were then subject to software thresholds $(\geq 3$ channels recording signals above $1 / 3$ photoelectron (p.e.) for S1 and a minimum area of $\sim 5$ ionisation electrons for S2). Only events with one $\mathrm{S} 1$ and one $\mathrm{S} 2$ were considered for further analysis. Of particular note here, $\chi^{2}$ goodness of fit indicators within the position reconstruction of both $\mathrm{S} 1$ and $\mathrm{S} 2$ were used to remove multiple-scatter events, and this was particularly effective for those with one vertex in a 'dead' region of the xenon, which would otherwise have been a troublesome background. Such 'dead' regions include the reverse-field volume between the cathode wire and the PMT grid wire [4] and the thin $(0.5 \mathrm{~mm})$ layer of xenon surrounding the PMT bodies. Double-Compton interactions with at least one vertex in these regions, referred to as 'multiple-scintillation single-ionisation' (MSSI) events, fulfil the previous selection criteria since there is no $\mathrm{S} 2$ pulse from the dead region and the coincident scintillation pulses are added together in a single S1. Unfortunately, perfecting this selection eventually required use of the full data-set as will be described in more detail below. 


\section{CALIBRATION}

\section{A. Scintillation Response and Position Reconstruction}

An external ${ }^{57} \mathrm{Co}$ source was inserted through the shield and located above the instrument every day. The dominant $122 \mathrm{keV} \gamma$-rays have a photoelectric absorption length of $3.3 \mathrm{~mm}$ in liquid $\mathrm{Xe}$, and hence provided good standard calibration candles from interactions close to the liquid surface. A typical ${ }^{57}$ Co spectrum is shown in Figure 2, The S1 signal channel exhibited a light detection efficiency at our operating field $(3.9 \mathrm{kV} / \mathrm{cm})$ of $L_{y}=1.8$ p.e. $/ \mathrm{keVee}$, decreasing from 5.0 p.e. $/ \mathrm{keVee}$ on application of the electric field. The $122 \mathrm{keV}$ interactions were used for a number of purposes to calibrate the instrument. Using S2 pulses, an iterative procedure, whereby a common cylindrical response profile was fitted to each channel, was used to normalise the measured response from each PMT (i.e. 'flat-field' the array). Position reconstruction in the horizontal plane was then achieved by using the converged response profiles in a simultaneous least-squares minimisation to all channels 7]. This method complements the Monte Carlo template matching procedure also being used but is less dependent on accurate iterative simulations [8]. Finally, the integrated areas of the $\mathrm{S} 1$ and $\mathrm{S} 2$ responses gave light collection correction factors as a function of radial position. Using this procedure a full-volume energy resolution of $\sigma=5.4 \%$ at $122 \mathrm{keV}$ was obtained with an energy reconstruction using a combination of the S1 and S2 responses to reflect the fact that, for electron recoils, these two channels are anti-correlated at a microscopic level. The individual $\mathrm{S} 1$ and $\mathrm{S} 2$ resolutions at $122 \mathrm{keV}$ are $16.3 \%$ and $8.8 \%$, respectively. Also shown in Figure 2 is the comparison of the response to simulation. Not only are the two main ${ }^{57} \mathrm{Co}$ lines well fitted but there is also a good match to the predicted Compton feature at $\sim 35 \mathrm{keV}$. The excess above $150 \mathrm{keV}$ is mainly due to the unsubtracted background. The left-hand panel in Figure 3 shows the distribution in the $x-y$ plane of events seen from the source. As expected most events are located towards the centre (the offset is due to an offset source position) with a radial fall-off as expected from the increasing thickness of copper along the line of sight.

\section{B. Stability, Electron Lifetime and Detector Tilt}

The ${ }^{57} \mathrm{Co}$ daily calibrations were used to assess the evolution of other operational parameters over the entire run: i) the average light and ionisation yields, as measured by fits to the ${ }^{57} \mathrm{Co} \mathrm{S} 1$ and $\mathrm{S} 2$ pulse area spectra; ii) the mean electron lifetime in the liquid, obtained from the exponential depth dependence of the ratio of the areas of the S2 and S1 signals (hereafter simply referred to as $S 2 / S 1$ ); iii) the evolution of the long-term detector

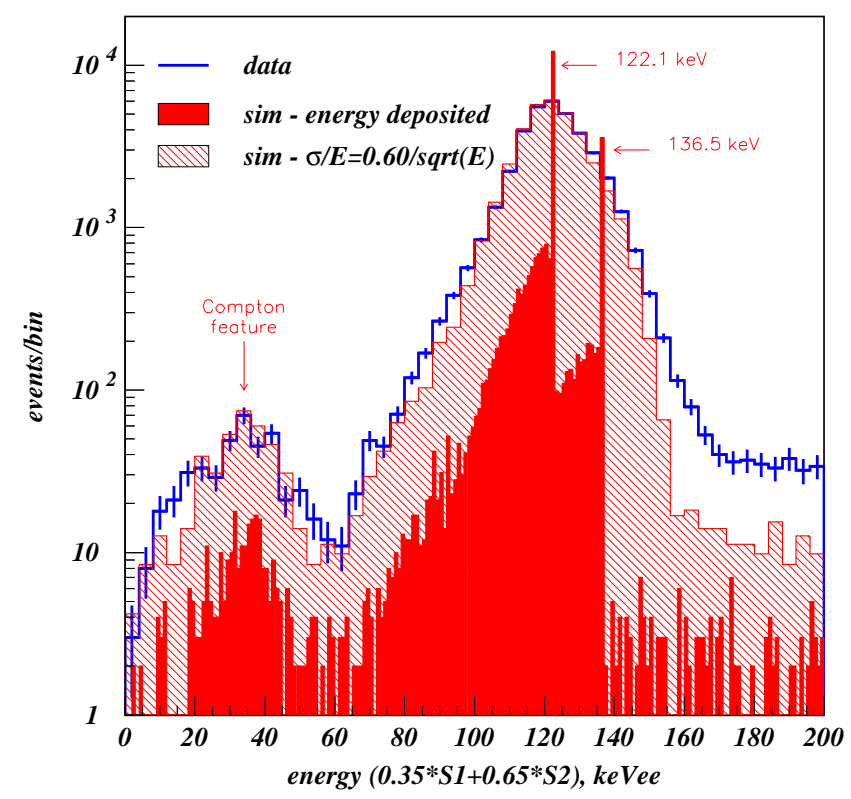

FIG. 2: Response to an external ${ }^{57} \mathrm{Co} \gamma$-ray source in the combined energy channel, exploiting S1 and S2 anti-correlation. One day's experimental data are shown in blue with statistical error bars. The simulation result is indicated in red: the solid histogram shows the bare energy deposits and the shaded one shows the result of Gaussian-smearing with the energy resolution indicated in the figure.
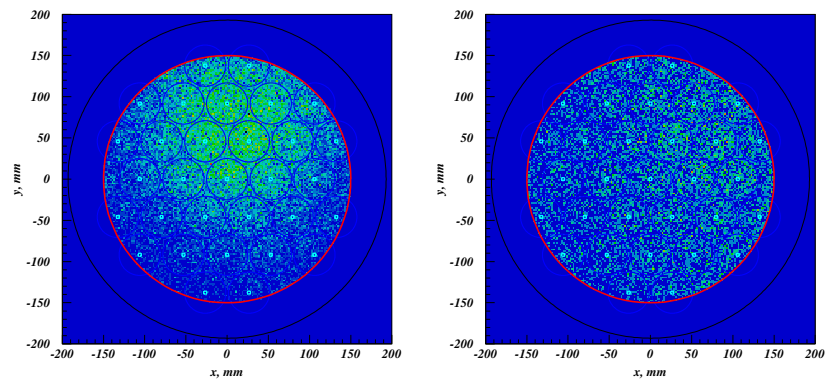

FIG. 3: Distribution in the horizontal plane of events from the ${ }^{57}$ Co source on the left and from the AmBe source on the right. The source positions are different for each image and neither is centred. In both cases the volume distribution is as expected from Monte Carlo simulations, given the location of each source. Interaction vertices can be seen out to the edge of the fiducial volume at a radius of $150 \mathrm{~mm}$ (red circle). The outer circle shows the edge of the liquid xenon target. Each PMT is marked by two smaller circles (PMT centres and envelopes).

tilt due to local geological factors, as given by the polar dependence of the S2-width distribution, which probes the thickness of the gas layer. The detector tilted by less than $1 \mathrm{mrad}$ over the run. Over the fiducial volume this corresponds to a systematic change in the gas gap of $<3.5 \%$, which in turn translates proportionally 
into a variation in the S2 signal. This was not deemed sufficient to warrant a full correction [31]. The scintillation mean light yield remained stable to a few percent, as did the ionisation yield, after correcting for the electron lifetime in the liquid. Remarkably, the lifetime did show an evolution during the run in the form of an improvement: from an initial value of $20 \mu \mathrm{s}$, achieved by initial gas-phase purification through external getters, a value of $35 \mu$ s had been reached by the end of the run (the full drift length of the chamber is only $14 \mu \mathrm{s}$ ). There was no active recirculation used and this improvement is attributed to the clean, xenon-friendly materials used in detector construction and to the uninterrupted application of the electric fields during the entire run. As the area ratio $S 2 / S 1$ is the main discriminant between nuclear and electron recoils, a depth-dependent correction must be applied to the S2 area to compensate for electron trapping by impurities. The electrons from the deepest events within the fiducial volume drifted for $13 \mu$ s and the correction factor for these varied from 1.92 at the start of the run to 1.45 at the end. The daily ${ }^{57}$ Co calibrations allowed this to be monitored throughout the science run and events were corrected individually using an historical trend profile.

\section{Linearity}

The linearity of the response of each channel in the array was investigated using low-energy Compton-scattered events from the ${ }^{137} \mathrm{Cs}$ source, in order to rule out hardware and software distortion for processing of small signals. The position of the vertex for each interaction was found and the waveforms from PMTs located a certain distance away from the vertex were selected based on the expected number of S1 photons, given the cylindrical response profile determined from the ${ }^{57} \mathrm{Co}$ data as pointed out in IA. Provided that the expected number is indeed small, the mean of the Poisson distribution for the number of detected photons can be quite accurately determined by counting the fraction of waveforms which do not contain any identified pulses, i.e. the frequency characterising the absence of any signal. This assertion is made against a sample of pure noise in the same waveform. Repeating this procedure for all channels and a range of expected signal allowed comparison of the mean $\mathrm{S} 1$ pulse area recorded in each trial against the expected Poisson mean, as shown in Figure 4 for the central PMT. In addition, this provides a very robust method to obtain the mean size of one photoelectron [9]. This has been calculated for every PMT within the array: the relationship is found to be linear to within the statistical accuracy of the measurement over a factor of 10 in mean pulse area, which covers the range of interest for WIMP nuclear recoil signals. The slope of the line in Figure 4 provides a measure of the mean single photoelectron response (SER) for that PMT. The mean SER of all the PMTs in the array has been found in this way to be in

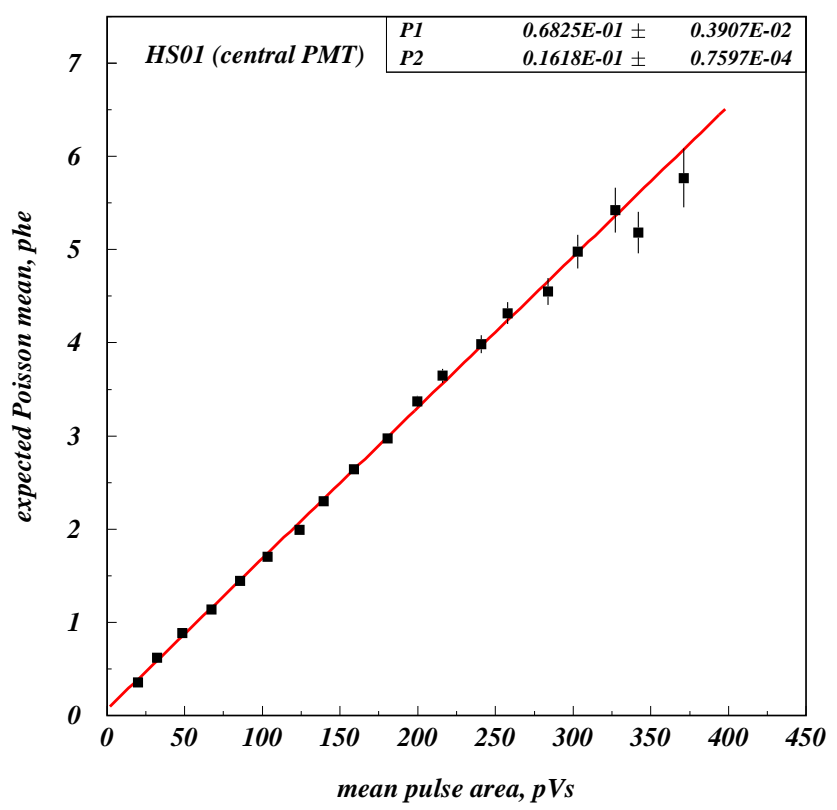

FIG. 4: Expected mean number of S1 photoelectrons as a function of the mean pulse area observed in the central channel in the array. The expected signal is the mean of the Poisson distribution obtained by counting the frequency of 'zeros', i.e. the absence of any response.

the range $47 \pm 12 \mathrm{pVs}$. The spread in these values forms part of the 'flat-field' correction discussed earlier; other dominant factors are the PMT quantum efficiency and imperfect hardware equalisation.

\section{Nuclear Recoil Response}

The nuclear recoil response in the energy range of interest to WIMP signals has been calibrated with neutrons from an $\mathrm{AmBe}(\alpha, \mathrm{n})$ source. The source was placed inside the polypropylene shielding above the detector but displaced to one side to reduce the interaction rate. The right-hand panel in Figure 3 shows the reconstructed event positions from the second calibration performed just after the science run had been completed. The distribution is slightly non-unform in the $x-y$ plane as expected.

Figure 5 shows a 'scatter-plot' of $\log _{10}(S 2 / S 1)$ as a function of energy in keVee from the AmBe calibration. The red line shows a smooth fit to the median of the elastic scatter distribution with $\pm 1 \sigma$ boundaries as blue lines. To obtain these curves the data were histogramed into 1 keVee bins and fitted by log-normal distributions. Examples of the quality of the fits are shown in Figure 6. The other well defined population in Figure 5, between 40-70 keVee, is due to inelastic scattering of neutrons from ${ }^{129}$ Xe nuclei and the more diffuse horizontal population is caused by associated $\gamma$-ray interactions. The 


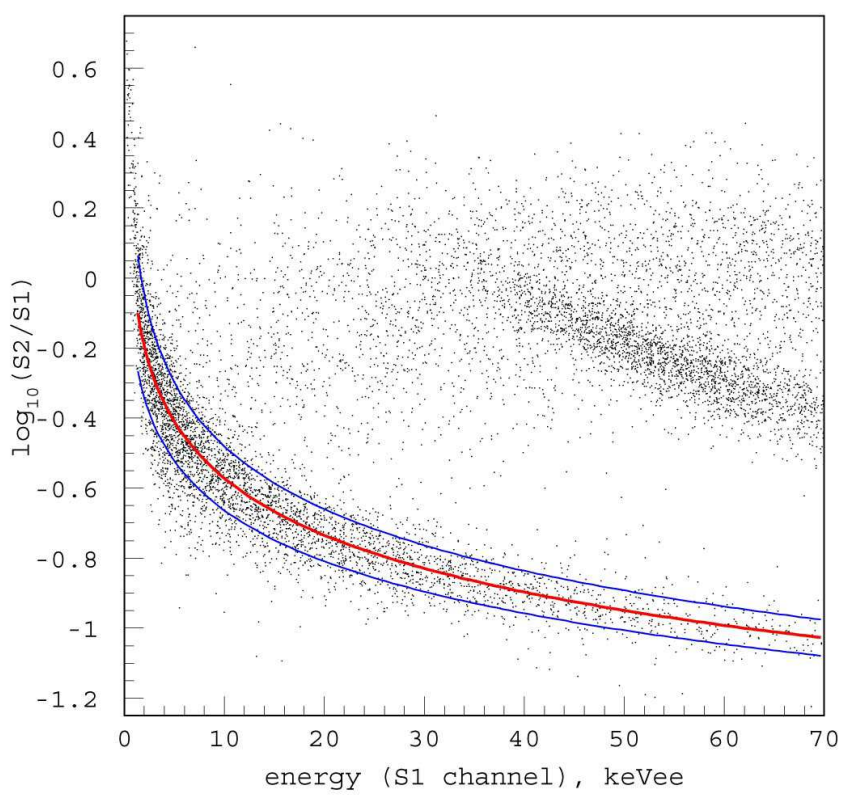

FIG. 5: Calibration of the nuclear recoil response with an AmBe neutron source, plotted as the discrimination parameter $\left(\log _{10}(S 2 / S 1)\right)$ as a function of 'electron-equivalent energy' (i.e. using the $\mathrm{S} 1$ channel calibrated by ${ }^{57} \mathrm{Co}$ ). The lines show the trends of the mean and standard deviation of energy-binned log-normal fits to the recoil population. The distinct population above $\sim 40 \mathrm{keVee}$ is due to inelastic neutron scattering off ${ }^{129}$ Xe nuclei.

elastic nuclear recoil median turns out to be very closely approximated by a power law, which is shown most effectively by replotting the figure in log-log form (Figure 7). Not only is the power-law behaviour very apparent but it can also be seen that there is less obvious flaring at lower energies than seen in other xenon experiments whose data were taken at much lower electric fields [2, 10]. Also shown are lines illustrating the approximate thresholds for S1 and S2.

\section{E. Electron Recoil Response}

The electron recoil response at low energies was established using a long duration calibration with a ${ }^{137} \mathrm{Cs}$ radioactive source. Compton scattering of the $662 \mathrm{keV} \gamma$-rays produced a significant number of events down to $\sim 2$ keVee but with only a small number extending far enough down in the $S 2 / S 1$ parameter to reach the nuclear recoil median (Figure 8). The general behaviour of the electron recoil band is reminiscent of the XENON10 results [10, 11, 12], but with a slightly more pronounced upturn at low energy, a larger separation between electron and nuclear recoil bands and narrower distributions. The low-energy electron-recoil populations in the ${ }^{137} \mathrm{Cs}$ and the WIMP-search data-sets were fitted in 1 keVee bins by a skew-Gaussian function. The fits were performed using a maximum likelihood (ML) method with a Poisson distribution as estimator for the observed data. Three of the fits are shown in Figure 6 , The distribution parameters are consistent bin-by-bin for the ${ }^{137} \mathrm{Cs}$ and WIMP data-sets, as confirmed in Figure 8. However, there are two distinct differences in the general behaviour. Firstly, the mean of the ${ }^{137} \mathrm{Cs}$ data is systematically lower than that of the WIMP data. It has been shown that this reduction is due to the high count rate used in collecting the Cs data causing the gain of the PMTs to be slightly suppressed due to saturation effects at low-temperature [9]. However, it was not feasible to lower the rate and still acquire sufficient data in a reasonable time and uncontaminated by other background. Secondly, the behaviour of the ${ }^{137} \mathrm{Cs}$ data-set in the low $S 2 / S 1$ tails is not closely representative of the science data, with the former exhibiting significantly more outliers. These events are attributed to MSSI double-Compton events as had been anticipated in [3]. This is not evident in Figure 8 as the number of events concerned was not sufficient to affect the standard deviation noticeably.

Double-Compton events in which both vertices are within the active volume produce two primary signals which are time coincident, but separated in position, and two secondary signals which are separated in both time (delay) and position. Even if they cannot be separated they are of no consequence as the combined ratio of $S 2 / S 1$ will be relatively unaffected. However, if one of the vertices occurs in a position from which no secondary is possible, then the only way to identify them is through positional mismatch between S1 and S2 and a less well reconstructed position from $\mathrm{S} 1$ as this has two vertices. If the 'dead' vertex is very close to one of the PMT surfaces the S1 signal can also appear to be too peaked within the array. Although there were already specific software cuts designed to deal with these events, some with certain topologies were not being fully identified by our analysis at that stage. For the ${ }^{137} \mathrm{Cs}$ data this problem was most apparent in the region $\log _{10}(S 2 / S 1)<-0.5$ and $E>30$ keVee but extended right down to the lowest energies. The ${ }^{137} \mathrm{Cs}$ calibration data were thus used to improve our algorithms for identifying MSSIs and the new routines were implemented after the science data had been opened. However, even with the improved selection cuts it was still not possible to use the ${ }^{137} \mathrm{Cs}$ data to predict accurately the expected number of single-scatter events leaking into the nuclear recoil region. The combination of lowering of the band mean (due to the rate dependent PMT sensitivity suppression at low temperature) towards the nuclear recoil band and remaining additional events in the lower wing caused a large overprediction of event leakage into the WIMP search box (41 events were predicted). The additional events remaining in the lower wing were probably due to the ${ }^{137} \mathrm{Cs}$ source not accurately mimicking that of the background sources due to its location. Hence, instead of using the ${ }^{137} \mathrm{Cs}$ 

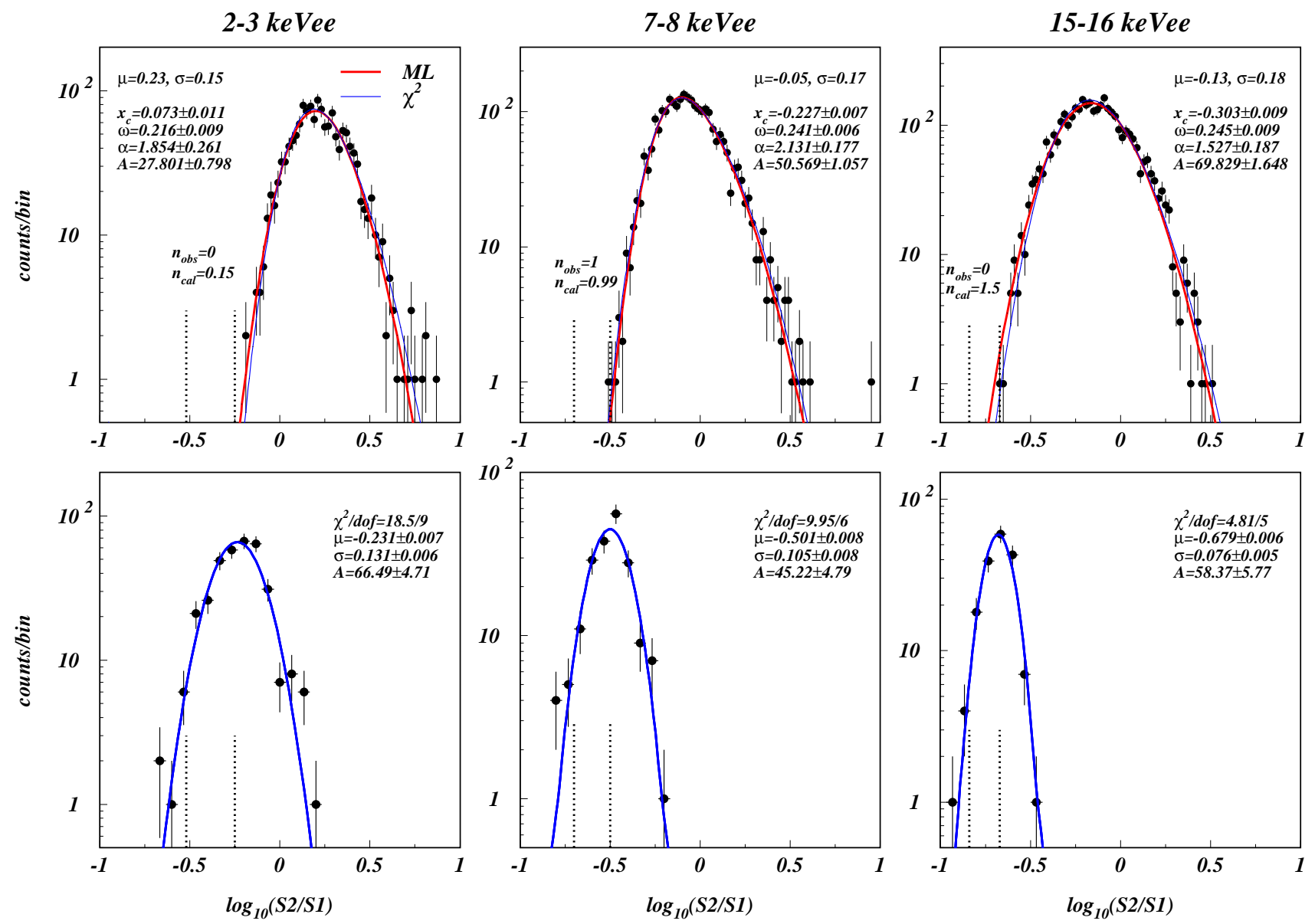

FIG. 6: Statistical fitting of the electron and nuclear recoil populations using the WIMP-search (upper panel) and AmBe datasets (lower panel). Three 1-keVee wide bins are shown: lowest, intermediate and highest energies accepted. The electron recoil population was fitted with a skew-Gaussian function using both the minimum $\chi^{2}$ (thin blue line) and a maximum likelihood (ML) method with the Poisson distribution as estimator (thick red line). The latter fit is more appropriate to data with low statistics (including zeros) in the tails of the populations as it uses a Poisson distribution as an estimator. Note that the entire population can be fitted (all energy bins, across the entire $\log _{10}(S 2 / S 1)$ range). The ML best fit parameters are indicated, along with the mean and standard deviation of the skew-Gaussian. The lower panels show the log-normal fits to the AmBe recoil data, which is used to define the acceptance region $[\mu-2 \sigma, \mu]$, between the vertical dashed lines. The number of electron recoils observed to be leaking into this region, $n_{\text {obs }}$, is compared with the estimated number, $n_{\text {cal }}$, from the ML fits. The total number of events expected in the acceptance region is $11.6 \pm 3.0$.

data, the WIMP-search data themselves were used to estimate the expected electron-recoil backgrounds, and this gave $11.6 \pm 3.0$.

\section{ANALYSIS OF THE WIMP SEARCH DATA}

\section{A. Data processing and selection}

The raw data were reduced using the purposedeveloped code ZE3RA (ZEPLIN-III Reduction and Analysis). The DAQ hardware records the 62 waveforms at $500 \mathrm{MS} / \mathrm{s}$ (2 ns samples) for $36 \mu$ s periods. ZE3RA finds candidate pulses in individual waveforms by searching for $3 \mathrm{rms}$ excursions above the baseline. Subsequent waveform processing includes resolving adjacent/overlapping pulses and grouping of statistically consistent structures (e.g. scintillation tails). A statisticallymotivated timing/shape coincidence analysis was then used to correlate occurrences on different channels thus allowing further pulse interpretation (e.g. clustering, identification of random coincidences, etc.) The resulting pulses were ordered by decreasing area in the highsensitivity (HS) sum channel and the largest 10 were stored in databases for further analysis. By design, ZE3RA does not ascribe physical meaning to pulses, it rather parameterises them in terms of arrival time, width, area, amplitude, etc. An event browser allows vi- 


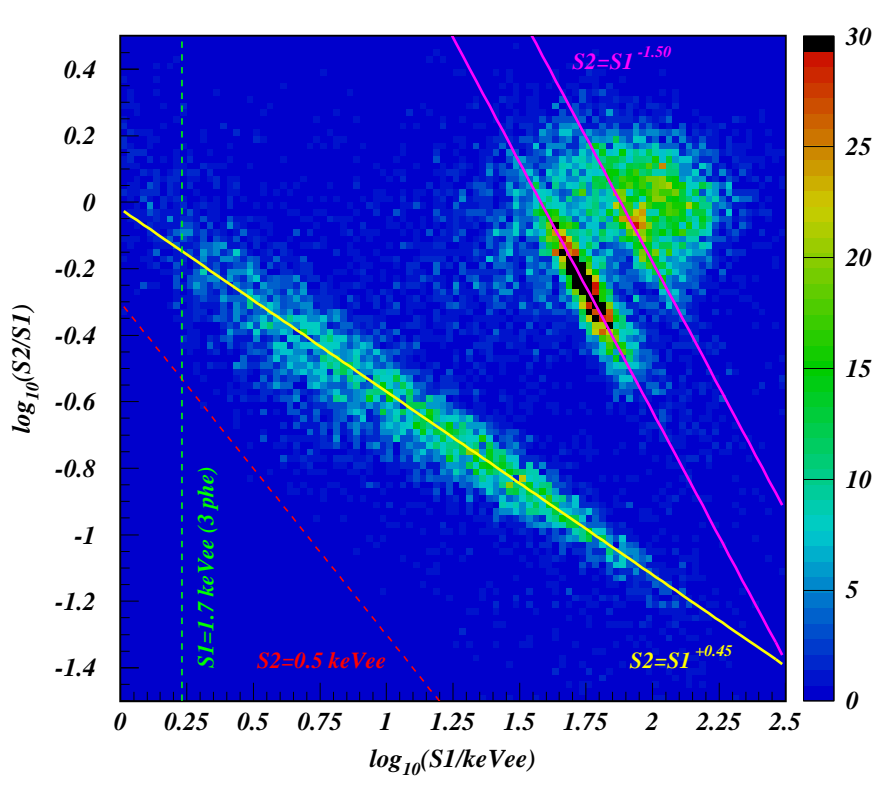

FIG. 7: Double-logarithmic plot of Fig. 5 showing the nuclear recoil population obeying the power-law trend indicated by the yellow line; the behaviour of the inelastic line from ${ }^{129} \mathrm{Xe}$ is markedly different, as this is dominated by charge recombination of the $40 \mathrm{keV} \gamma$-ray rather than the small $\mathrm{nu}$ clear recoil component of the deposited energy. Approximate thresholds for S1 (3-fold software trigger) and for S2 (hardware trigger) are also indicated. From this it can be seen that the S2 hardware trigger corresponds to $\mathrm{S} 1=0.5 \mathrm{keVee}$ for nuclear recoils.

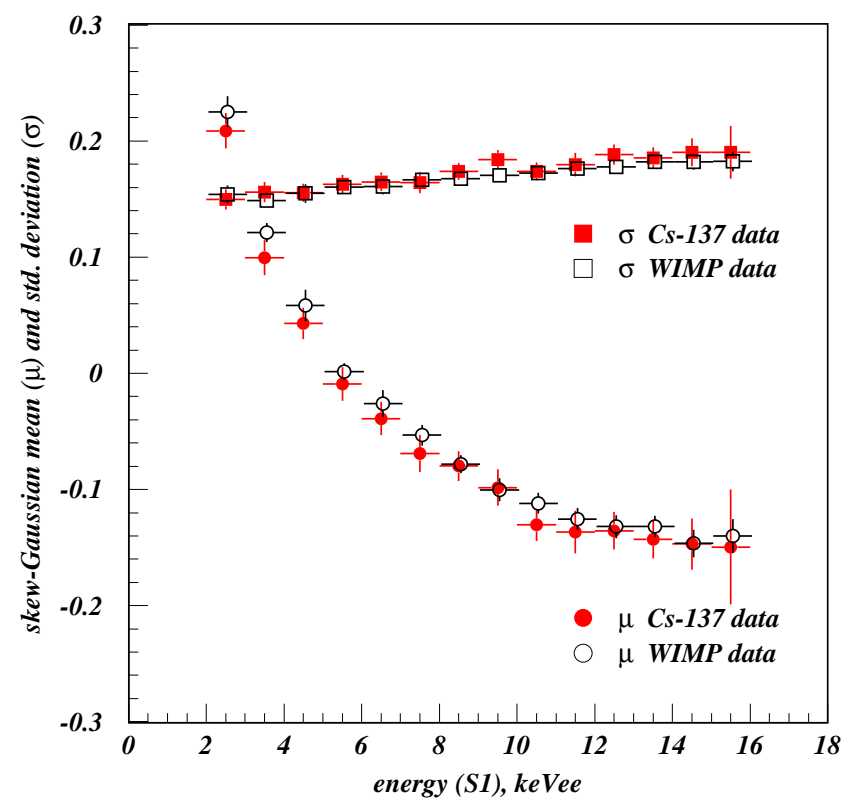

FIG. 8: Comparison of the skew-Gaussian mean and standard deviations for the ${ }^{137} \mathrm{Cs}$ and WIMP-search data-sets calculated from the ML-fit parameters (the horizontal error bars indicate the bin width). sual scanning of events, channels or individual pulses; a batch-mode interface allows scripted reduction of large data-sets.

The data structures produced by ZE3RA were analysed by a flexible code based on hbook [13]. It processed the original parameters to assign physical meaning to pulses in events according to a well defined set of rules (e.g. primary scintillation signals are fast and must precede wider electroluminescence signals). Only events that can represent single scatters in the two-phase target ('golden' events with one S1 and one S2) were retained. Primary (S1) pulses were found by applying an acceptance threshold of $1 / 3$ p.e. to the ZE3RA pulses and also requiring a 3 -fold coincidence amongst the 31 PMTs. This software threshold was nominally equivalent to an energy threshold of $1.7 \mathrm{keVee}$. Exceptions in the S1 selection were allowed for PMT after-pulses. These are signal-induced artifacts generated within the PMTs. In general they have a characteristic time delay from the optical signal, but with a wide distribution and, moreover, it varies between PMTs. As a result it is not trivial to identify after-pulsing and avoid them instead being classified as additional S1 signals, which would result in the event being wrongly rejected. Secondary (S2) pulses were required to have at least an integrated area corresponding to the signal expected from about 5 electrons leaving the liquid surface. This suppresses optically-induced singleelectron emission [22] as well as optical feedback effects from the cathode grid, which are not part of the direct measure of the ionisation signal generated at the interaction site. Many additional parameters are derived for these, such as 3-D position information, hit-pattern descriptors, interaction energy and corrections (e.g. array flat-fielding, electron lifetime, liquid level, light collection, etc). Subsequent analysis (science exploitation) is based on PAW [14] and ROOT [15].

Trapping MSSI events effectively was a significant challenge, involving a combination of approaches: use of goodness of fit indicators in the position reconstruction algorithms, comparison of coordinates derived independently from S1 and S2, and searching for abnormal light patterns across the array.

\section{B. The WIMP Search Box}

Discrimination between nuclear and electron recoils is illustrated in Figure 9 which combines electron recoil data from ${ }^{137} \mathrm{Cs}$ and elastic nuclear recoil data from AmBe. The separation between the two populations is clear and this is used as the main way of defining the nuclear recoil search box for potential WIMP events. The selection cuts used can be categorised as follows:

1. Golden event selection (including pulse finding, S1 and S2 definition, and single scatter selection)

2. Waveform quality cuts (mild cuts mainly aimed at 


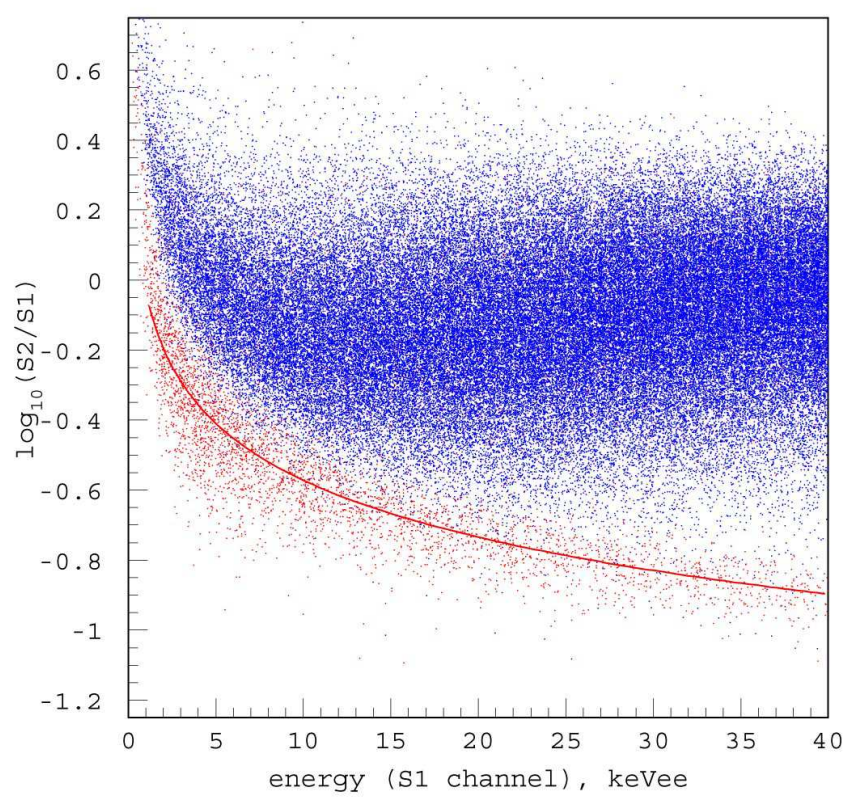

FIG. 9: Combined scatter plot of $\log _{10}(S 2 / S 1)$ as a function of energy from the two calibration data-sets, ${ }^{137} \mathrm{Cs}$ and $\mathrm{AmBe}$. The upper population corresponds to low-energy Compton electrons and the narrower, lower one to nuclear recoils produced by neutron elastic scattering.

large baseline excursions compromising pulse parameterisation)

3. Pulse quality cuts (mild cuts to avoid extreme outliers in parameter distributions)

4. Fiducial volume definition (drift time window and a radial limit from the $\mathrm{S} 2$ position reconstruction)

5. Event quality cuts (strong cuts to deal with MSSI events mainly)

The fiducial definitions (4) leave an active mass of $6.52 \mathrm{~kg}$ with a raw exposure of $453.6 \mathrm{~kg}$.days. Low-energy events in the $10 \%$ data were well separated from the nuclear recoil median line down to the lowest energies. The WIMP search box boundary was thus defined as $2<E<16$ keVee and $\left(\mu_{n}-2 \sigma\right)<\log _{10}(S 2 / S 1)<\mu_{n}$, where $\mu_{n}$ is the energydependent mean of the nuclear recoils (acceptance of $47.7 \%$ ). This region was defined before unblinding and was kept for the subsequent analysis. The effective total exposure within this box, after taking account of all of the efficiencies, as detailed in Table \, is $127.8 \mathrm{~kg} \cdot$ days.

\section{Backgrounds}

Electron and nuclear recoil background predictions for ZEPLIN-III are based on a full GEANT4 [16] simulation including measured radioactive content levels for all major components [3]. The largest contributor, by far, is

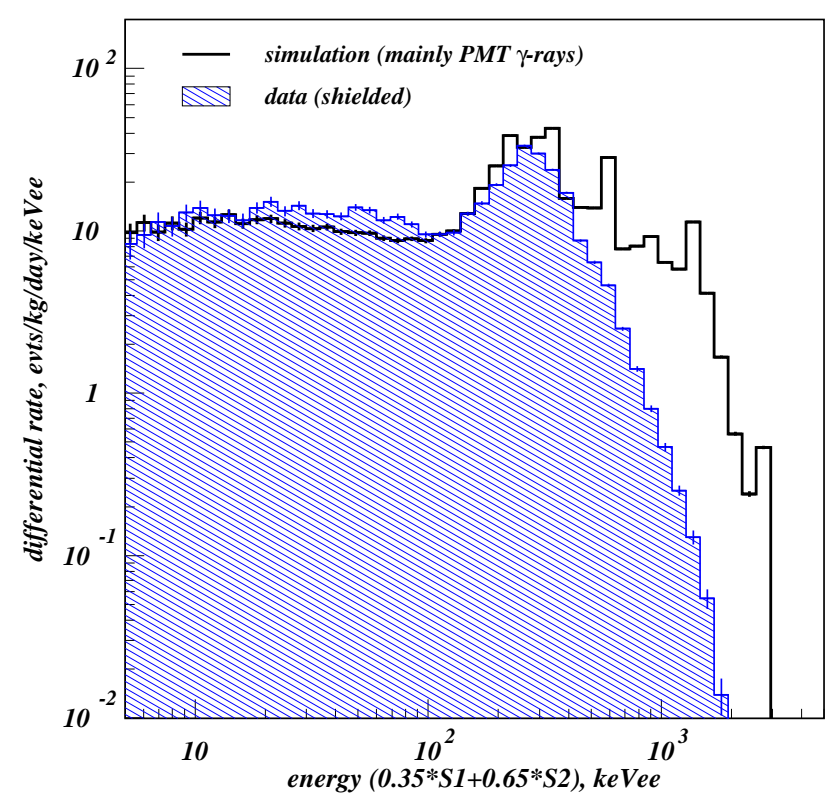

FIG. 10: Electron recoil background measured during the fully-shielded science run. The differential spectrum is shown superimposed on the Monte Carlo prediction [3] using GEANT4 [16] without rescaling. The latter includes a dominant 10.5 evts $/ \mathrm{kg} /$ day $/ \mathrm{keVee}$ ('dru') from the photomultipliers, $\gamma$-rays from the lead 'castle' $(0.7 \mathrm{dru}), \beta$-particles from ${ }^{85} \mathrm{Kr}(0.2 \mathrm{dru})$ and $\gamma$-rays from ceramic feedthroughs (0.1 dru). The disagreement at high energies is caused by single-scatter selection in the data (but not in the simulation) and by the limited DAQ dynamic range which was optimised for the WIMP-search run.

the PMT array. Figure 10 shows the measured differential background spectrum together with the simulated background. The high-energy region above $300 \mathrm{keVee}$ is suppressed due to dynamic range limitation.

The expected single-scatter neutron background in the data-set is $1.2 \pm 0.6$ in the WIMP search box with $90 \%$ coming from PMT generated events through $(\alpha, \mathrm{n})$ interactions and spontaneous fission. The remaining $10 \%$ are mainly from contaminants in ceramic feedthroughs and external leakage through the shield of neutrons from the rock.

\section{WIMP Signal Search}

Figure 11 shows the final scatter plot from the complete science data-set. There are 7 events within the WIMP search box and the energy scale is shown in keVee. To assess the implications of these events the energy scale needs to be converted into keVnr, the energy dependent detector efficiency for nuclear recoils must be found and the relative likelihood of any of those 7 events being drawn from the expected WIMP distribution rather than the extended electron-recoil distribution must be calcu- 


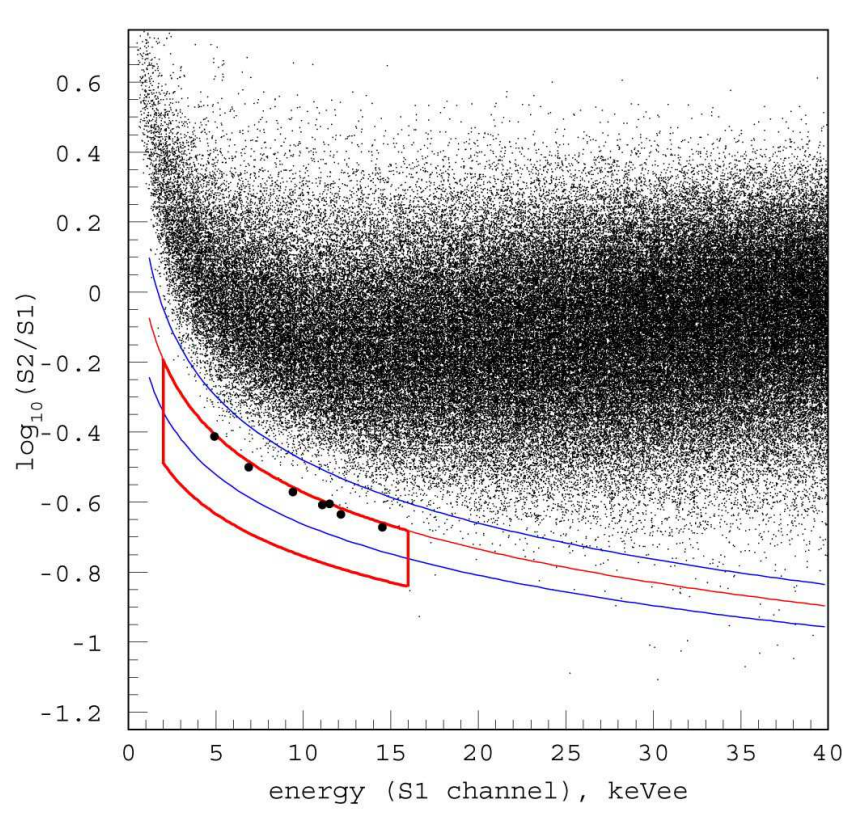

FIG. 11: Scatter plot of $\log _{10}(S 2 / S 1)$ as a function of energy for the entire 83-day data-set of first science run. There are 7 events (large dots) in the WIMP-search region (thick red box), which extends from $2<E<16$ keVee and $\mu_{n}-2 \sigma_{n}<$ $\log _{10}(S 2 / S 1)<\mu_{n}$, where $\mu_{n}$ is the energy-dependent mean of the nuclear recoils (thin red line bordered by the blue curves at $\left.\pm 1 \sigma_{n}\right)$. These are all located near the upper boundary, between $\simeq 5-15 \mathrm{keVee}$.

lated.

The level of discrimination apparent in Figure 11 is very high. As derived from the data themselves, the average $\gamma$-ray rejection factor is $5 \times 10^{3}$ between $2-16 \mathrm{keVee}$ with an increase below 5 keVee. This is significantly better than had previously been demonstrated by the XENON10 experiment which achieved $99.9 \%$ at the very lowest energies [10] whilst our data exhibit better than $99.99 \%$ in the $2-5$ keVee band.

Figure 12 shows the spatial $x-y$ distribution of all events in the 2-16 keVee energy range. Events within the WIMP search box are highlighted.

To derive the significance of the events within the search box the experiment efficiency must be derived together with the energy scale conversion between keVee and keVnr. These are established in the following sections. First the efficiency for nuclear recoil detections is found by comparing AmBe data-sets with very different trigger thresholds in both hardware and software. The energy scale conversion is then done by comparing a simulation of the expected nuclear recoil with that measured.

\section{Efficiency and threshold}

The overall detection efficiency will be a combination of hardware and software effects. As mentioned earlier

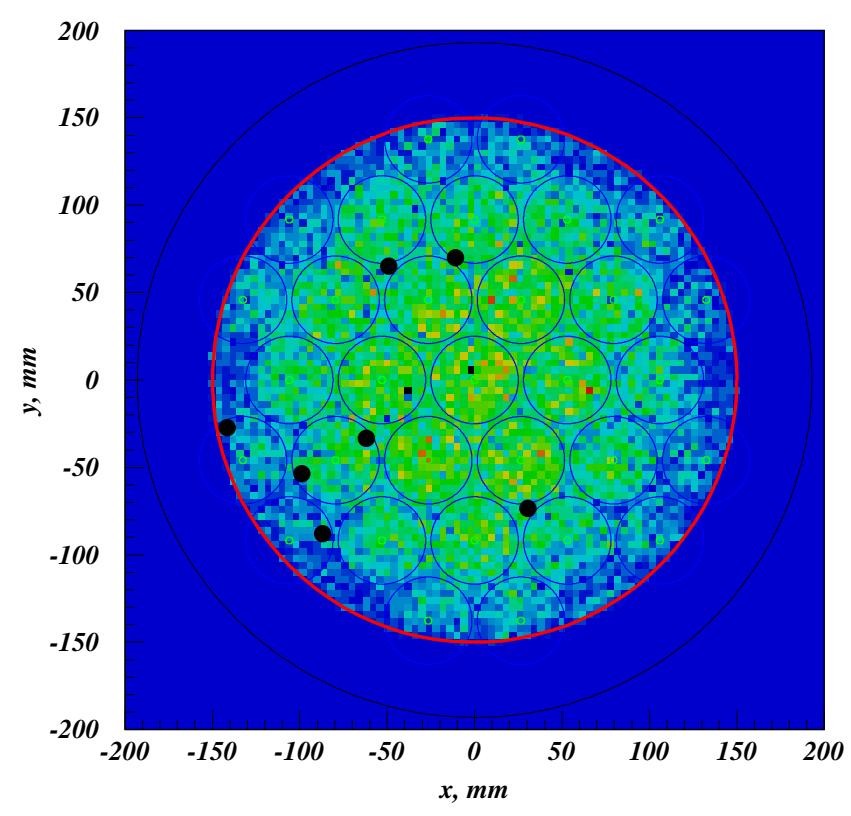

FIG. 12: Horizontal distribution of events in the energy range 2-16 keVee for the science run data-set produced in the same way as those in Figure 3 The reconstructed location of the 7 events in the acceptance region is indicated. The measured distribution of the overall background is consistent with detailed Monte Carlo simulations of that expected from the instrument activity which is dominated by the photomultipliers.

in Section \B the hardware trigger threshold is derived from S2 in the low-energy part of the S1 spectrum relevant to WIMP signals. At higher energies, well beyond the upper limit of the WIMP search box, there is a highlevel inhibit to suppress the overall count rate, but this does not affect the efficiency at low energy. Dead-time effects are usually energy independent. Software effects include thresholding associated with pulse finding algorithms and selection cuts. These have been described in Section [C] The energies (expressed in S1 keVee) at which these 'thresholds' will affect the detection efficiency are tabulated in Table I. In order to confirm these expectations a second $\mathrm{AmBe}$ data-set was analysed as a check on the energy dependence near the threshold. This data-set had been acquired with a lower hardware S2 trigger threshold. In addition, the 3-fold S1 coincidence requirement was changed to 2 -fold in this particular analysis and all quality cuts removed or significantly relaxed. The overall effect of these two changes is shown in Figure 13 by comparing the black histogram labelled 'Am-Be low-threshold data' with the blue shaded histogram labelled 'Am-Be calibration data'. The difference between these two histograms is only noticeable below $\mathrm{S} 1 \sim 4$ keVee as expected. A study of the smallest S2 events triggering the system in each run has shown directly that the trigger level in the two runs was $\sim 11$ and $\sim 4$ ionisation electrons, respectively. These numbers were calibrated against the 
measured single electron spectrum for ZEPLIN-III following the method already used for ZEPLIN-II [22]. The experiment efficiency during the science run is taken as the ratio of the two AmBe data-sets, shown in Figure 14

The full red curve labelled 'Simulation $($ Eee/Enr=2.09)' in Figure 13 shows a Monte Carlo simulation of the expected differential spectrum which should have been seen by the experiment assuming a constant ratio between $\mathrm{S} 1 \mathrm{keVee}$ and $\mathrm{S} 1 \mathrm{keVnr}$. This simulated curve has not been corrected for instrument efficiency but even so it is clear that there is a departure from the experimental data below $\mathrm{S} 1 \sim 20 \mathrm{keV}$. Given that this mismatch extends so far in energy above any reasonable thresholding effects, it is interpreted as evidence for a non-linear scale conversion.

\section{Energy conversion}

A comparison between the differential spectrum seen during the nuclear-recoil calibration, using AmBe, and a Monte Carlo simulation has been used to derive the energy scale conversion between keVee and keVnr. This relies on the integrity of the simulation using GEANT4, which is very well established in general for elastic scattering of neutrons, and which has been further extensively validated as part of this work. Systematic effects related to the simulation of the experimental calibration were assessed. These included, amongst others: variations in neutron source spectrum and source location inside the shield; the effect of intervening and surrounding materials; simulation event selection; energy resolution smearing; coincident AmBe $\gamma$-rays; treatment of inelastic scattering in xenon. The Monte Carlo result at low recoil energies was very resilient to sensible variation of these parameters. A different Monte Carlo code [17] confirmed these results independently. Naturally, incorrect angular cross-sections for elastic scattering off xenon could be invoked to explain the low-energy result, since enhancing forward scattering would soften the recoil spectrum. However, dedicated simulations confirmed the correct implementation of the ENDF/B-VI evaluated data libraries [18] which underpin the GEANT4 low-energy neutron transport models. Both angular and energydifferential cross-sections were found to be in agreement with ENDF/B-VI and similar databases. An implementation in GEANT4 of the more recent ENDF/B-VII data for xenon by the XENON10 team [12], aimed at exploring the causes of a similar effect observed by that experiment, found only minor differences in the recoil spectrum produced by a similar neutron source. We have independently confirmed this conclusion. The comparison between simulation and experiment for ZEPLIN-III is shown in Figure 13. The energy scale associated with the simulated data has been converted from keVnr to keVee in Figure 13 by simply dividing by 2.09 , to allow for the combination of the relative nuclear-recoil scintillation efficiency to that of a $122 \mathrm{keV} \gamma$-ray at zero electric

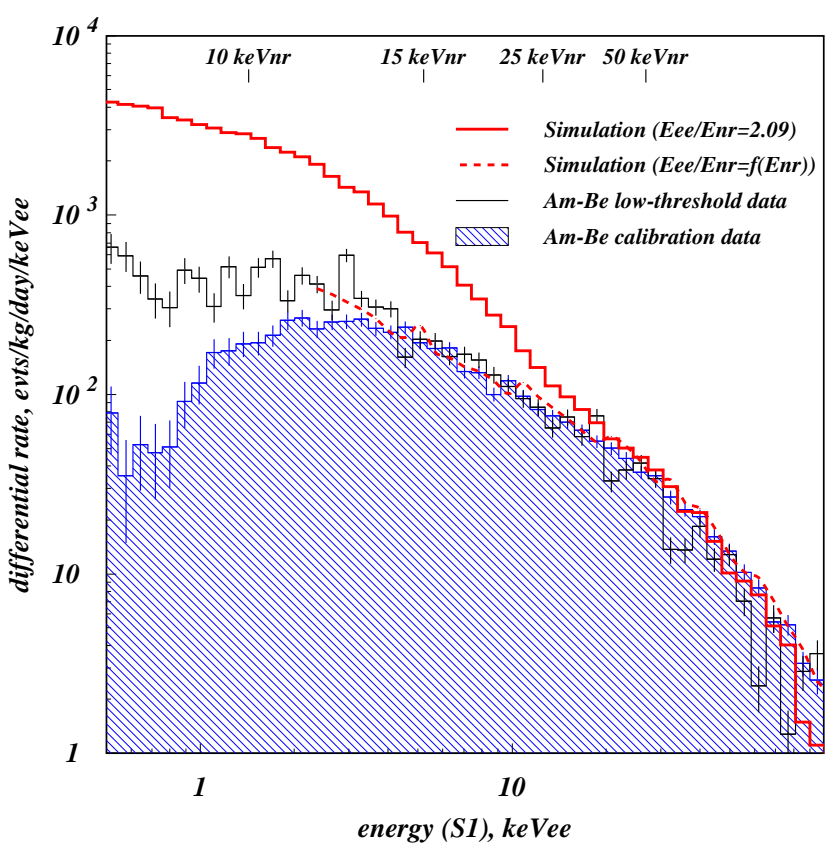

FIG. 13: Differential energy spectra for the AmBe elastic recoil population in $\mathrm{S} 1$ electron-equivalent units $\left({ }^{57} \mathrm{Co}-\right.$ calibrated S1). The main calibration data (shaded blue histogram) and the lower threshold data-set described in the text (black) are compared with the Monte Carlo simulation using a constant conversion factor between nuclear recoil and electron equivalent energies (solid red curve). The ratio between these two curves is interpreted as an energy-dependent efficiency factor and occurs in the low-energy region where thresholding effects are expected. The dashed red curve is the result of the nuclear recoil non-linearity analysis described in the text, which results in the energy conversion indicated by the markers at the top of the figure.

field, $L_{e f f}$, and a suppression factor, $S$, which allows for the field-dependent variation in the scintillation output. These are used in the following equation:

$$
E_{n r}=\frac{S 1}{L_{y}} \frac{S_{e}}{L_{e f f} S_{n}}
$$

where $S_{e}$ and $S_{n}$ are the suppression factors in the scintillation output for $122 \mathrm{keV} \gamma$-rays and nuclear recoils, respectively, at the experiment operating fields. Note that in this equation the ratio $S 1 / L_{y}$ defines the keVee unit. Above $E_{n r} \sim 20 \mathrm{keV}$ the available experimental data for $L_{e f f}$ suggests it is constant at $\sim 0.19[19,20$, 21]. However a variation in $L_{e f f}$ at low energy has been invoked to explain XENON10 neutron calibration data [12] and hence is allowed to vary in this work.

In general the conversion between an electronequivalent energy scale, in keVee, and a nuclear recoil energy scale, in keVnr, is not necessarily linear and any non-linearity can be expressed mathematically through energy dependency in $L_{e f f}$ and/or $S_{e} / S_{n}$. Above $E_{n r} \sim 20 \mathrm{keV}$ the available experimental data for $L_{\text {eff }}$ sug- 
TABLE I: Energy-independent efficiency factors and thresholds due to hardware and software actions. Efficiency figures are constant over the WIMP recoil range. Numbers following the entries refer back to the list of software operations itemised in Section ПIB The total effective exposure is $127.8 \mathrm{~kg} \cdot$ days.

\begin{tabular}{|l|c|l|}
\hline Effect & Efficiency & Method \\
\hline Deadtime & $91.7 \%$ & Measured \\
Hardware upper threshold & $100 \%$ & On-off compare \\
ZE3RA pulse finding (1) & $96.0 \%$ & Visual inspection \\
Event reconstruction (2,3) & $91.9 \%$ & Vand calculation \\
Selection cuts (5) & $73.0 \%$ & On-off compare \\
WIMP box acceptance & $47.7 \%$ & Calculation \\
\hline Effect & Threshold & Method \\
\hline Hardware (S2) trigger & $\mathrm{S} 1=0.5 \mathrm{keVee}$ & Two data-sets \\
& & Visual inspection \\
& & Modeling \\
Pulser tests \\
Software S2 area & $\mathrm{S} 1<1 \mathrm{keVee}$ & Calculation \\
Software S1 3-fold & $\mathrm{S} 1=1.7 \mathrm{keVee}$ & Calculation \\
& & Two data-set \\
analyses
\end{tabular}

${ }^{a}$ All thresholds are quoted here in terms of the S1 signal in keVee for nuclear recoils. The equivalent nuclear recoil energy, keVnr, depends on the conversion between keVee and keVnr. For the relationship shown in Section IIID2 11 ionisation electrons corresponds to $<7 \mathrm{keVnr}$

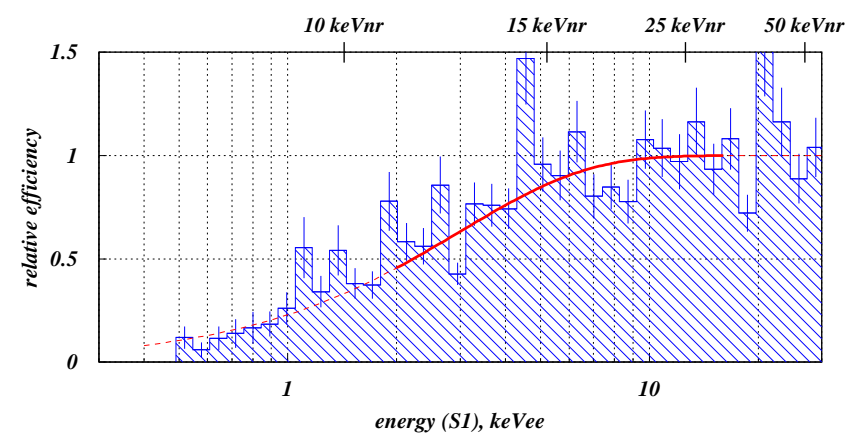

FIG. 14: Energy-dependent part of the nuclear recoil detection efficiency as deduced empirically by comparing the two experimental AmBe spectra shown in Fig. 13. The 'lowthreshold' run was taken with a lower hardware trigger threshold; in addition, software quality cuts were relaxed, along with the S1 3-fold requirement. A fit to the data is shown, with the WIMP acceptance box indicated by the thicker portion of the line. The S2 hardware trigger in the low-threshold AmBe run was half of that used in the science data-set and thus corresponded to $\mathrm{S} 1=0.25 \mathrm{keVee}$, and the $\mathrm{S} 1$ pulse finding algorithms only required a 2 -fold detection above $1 / 3$ p.e. giving a nominal software threshold of $\mathrm{S} 1=1.1 \mathrm{keVee}$. Hence above $\mathrm{S} 1=2 \mathrm{keVee}$ (the lower WIMP acceptance box boundary) the 'low-threshold' data-set has near-unity efficiency.

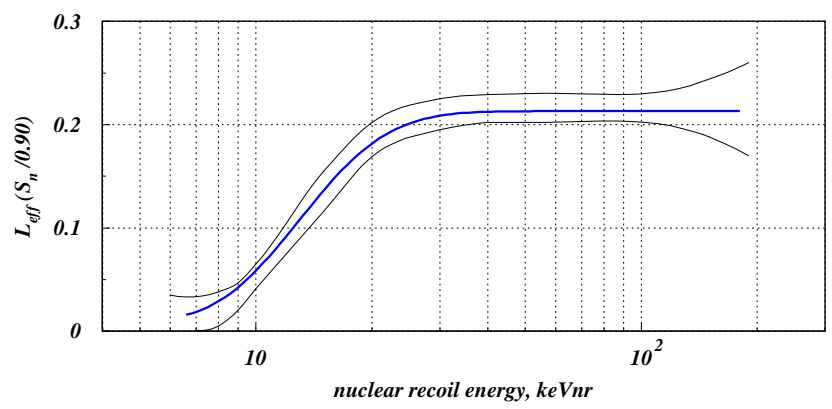

FIG. 15: The derived energy-dependent behaviour of $L_{e f f} \cdot S_{n}$. The thick curve shows the best fit to the data, but other curves producing very similar goodness-of-fit indicators are obtained within the envelope shown. The constraints become very weak outside the energy range shown.

gests it is constant at $\sim 0.19$. At lower energies the situation is much less clear 12. For $S_{n}$ there are no data on the energy dependence but rather there is a single value based on a measurement at $56 \mathrm{keVnr}$ using a neutron beam [20]. This gives $S_{n}=0.90$ at our field and it is commonly assumed to remain constant over the whole energy range of WIMP nuclear recoils. If $L_{\text {eff }}$ and/or $S_{n}$ are not constant below $\sim 20 \mathrm{keVnr}$ this will cause a non-linearity in the nuclear recoil energy scale.

In the following it is assumed that such non-linearities are responsible for the mismatch seen in Figure 13. The approach used is similar to that applied to the XENON10 data [12]. Using a maximum-likelihood technique we have derived a non-linearity function which best matches the AmBe simulation to our neutron calibration spectrum above $\sim 2$ keVee. The outcome of this process is shown as the dashed red curve in Figure 13. Figure 15. expresses the nonlinearity in terms of the combined effect of $L_{e f f}$ and $S_{n}$, with the latter referenced to 0.90. In Figures 13 and 14 the top horizontal axes show the energy scale in keVnr to be compared with keVee on the bottom scale. The WIMP search box boundaries then translate to 10.7 and $30.2 \mathrm{keVnr}$. One consequence of the required non-linearity is a marked reduction in efficiency for nuclear recoil detection below $15 \mathrm{keVnr}$.

\section{Limit analysis}

The event box contains a large empty region with a small number of events close to where a tail from the electron recoil distribution is expected. However, although there is a good fit of a skew-Gaussian distribution to the electron-recoil band above the WIMP search box, there remains systematic uncertainty about an extrapolation of this being used as an accurate estimator of the number of expected background events in the box. The fact that the best fit expectation exceeds the measured number of events might result in an artificially lower upper limit, as pointed out in [23]. This compromises any straight- 
forward use of maximum-likelihood techniques and even the commonly-used Feldman-Cousins (FC) analysis 23]. Hence, a simpler, more transparent and conservative approach is adopted based on a minimum of three pieces of information about the data.

The first is the reasonable assumption that any expected electron-recoil background will fall in the top part of the WIMP search box. Based on this assumption the box is divided into two regions which have significantly different probabilities of having electron-recoil background within them. This is done in Figure 16 after transforming the WIMP search box so that the vertical axis has a linear scale in nuclear recoil acceptance percentiles as derived from the AmBe calibration data. In this representation any WIMP nuclear recoil signal should populate the box uniformly, whereas the density of the electron recoil background is expected to decrease monotonically down from the top. A horizontal dashed line is shown which divides the WIMP search box into two regions such that the top area contains all the events. In the following analysis the fractional area in the lower region is denoted by $f$.

The second is the observation that no WIMP event is seen in the lower region $\left(n_{l}=0\right)$.

Finally, it is possible that there may be up to 7 WIMP events in the upper region $\left(n_{u} \leqq 7\right)$.

A classical $90 \%$ one-sided upper limit for the WIMP expectation value in the whole box, $\mu$, is the value under which $10 \%$ of repeated experiments would return zero events in the lower box and up to 7 in the upper box. This is expressed in terms of Poisson probabilities as

$$
\begin{aligned}
& P\left(n_{l}=0, n_{u} \leqslant 7 \mid \mu\right)= \\
& \quad P\left(n_{l}=0 \mid f \mu\right) * \sum_{i=0}^{7} P\left(n_{u}=i \mid(1-f) \mu\right)=0.1
\end{aligned}
$$

Over the range of values of $f$ between 0.75 and 0.84 the calculated result is $\mu=2.30 / f . f=0.84$ is the maximum area allowed which just excludes all of the events.

It turns out that, for the value of $\mu$ resulting from this calculation, the second factor in equation (2) is very close to unity regardless of the area fraction, $f$. This reflects the fact that the upper limit is driven almost entirely by the presence of the empty region and the value 2.30 is then recognised as the classical $90 \%$ upper limit on zero. It is then reasonable to assume that the two-sided $90 \%$ confidence interval for this particular data-set will also be driven by the empty box. In this case the upper limit to this interval will be at $\mu=2.44 / f$, with 2.44 being the corresponding 2-sided FC upper limit on zero 23]. Figure 16] shows a dividing line with $f=0.8$, which is adopted as a conservative boundary placement beyond which no background is likely. The $90 \%$ confidence interval upper limit is then $\mu=3.05$. With this extreme value of $\mu$ there is a $54 \%$ probability that there are indeed no WIMP events in the upper region, a $33 \%$ chance of there being 1 WIMP event and a $13 \%$ chance of $\geqq 2$ WIMP events. The fact that the most likely scenario is no WIMPs in

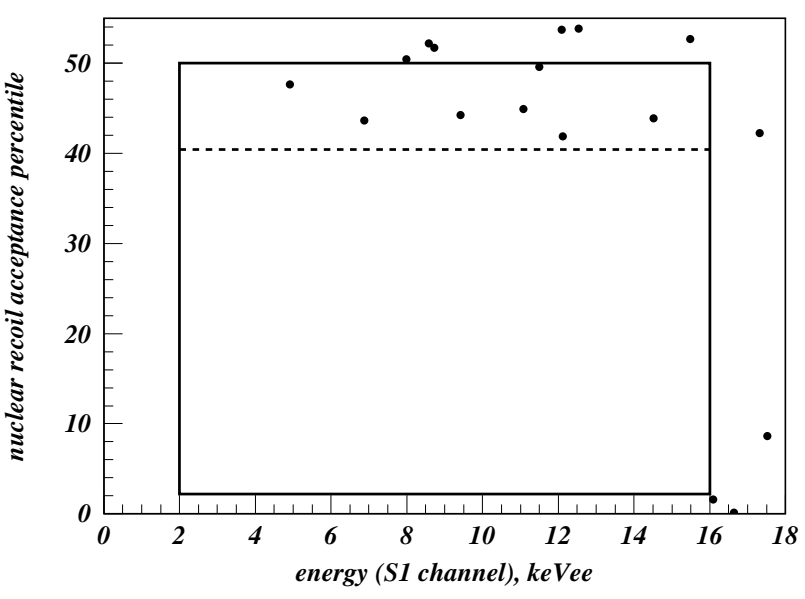

FIG. 16: The WIMP search box with the vertical axis remapped onto nuclear recoil percentiles. This is done using the $S 2 / S 1$ distribution from the AmBe calibration data. The positions of the 7 events falling within the box are shown as well as other events just outside the box. The horizontal dashed line separates the box into two regions with an area ratio of $1: 4$.

the data-set even with $\mu=3.05$ implies that $\mu=0$ is included within the $90 \%$ two-sided interval as the null event hypothesis becomes more and more likely as $\mu$ is reduced.

The upper limit of 3.05 events is used to derive the upper limit to the WIMP-nucleon spin-independent elastic scattering cross-section as a function of WIMP mass. The signal energy distribution is obtained from the theoretical WIMP recoil spectrum 24], derived using the standard spherical isothermal Galactic halo model $\left(\rho_{d m}=0.3 \mathrm{GeVcm}^{-3}, v_{o}=220 \mathrm{~km} / \mathrm{s}, v_{e s c}=600 \mathrm{~km} / \mathrm{s}\right.$ and $v_{\text {Earth }}=232 \mathrm{~km} / \mathrm{s}$ ), detector response efficiencies and energy resolution. The form factor is taken from [25]. The expected distribution in $S 2 / S 1$ is determined from the neutron calibration.

The final result for the $90 \%$ confidence interval upper limit to the cross-section, shown in Figure 17, has a minimum of $8.1 \times 10^{-8}$ pb for a WIMP mass of $60 \mathrm{GeV} / c^{2}$. In the mass range beyond $100 \mathrm{GeVc}^{-2}$ this result complements the XENON10 result and further constrains the favoured SUSY parameter space 26] from xenon-based experiments. Spin-dependent limits are presented separately [29].

\section{CONCLUSIONS}

An analysis of $847 \mathrm{~kg} \cdot$ days of data from the first science run of ZEPLIN-III has resulted in a signal lower limit consistent with zero, and an upper limit on the spin-independent WIMP-nucleon elastic scattering crosssection of $8.1 \times 10^{-8} \mathrm{pb}$, at $90 \%$ confidence level. In reaching this result it was necessary to confront an unexpected 


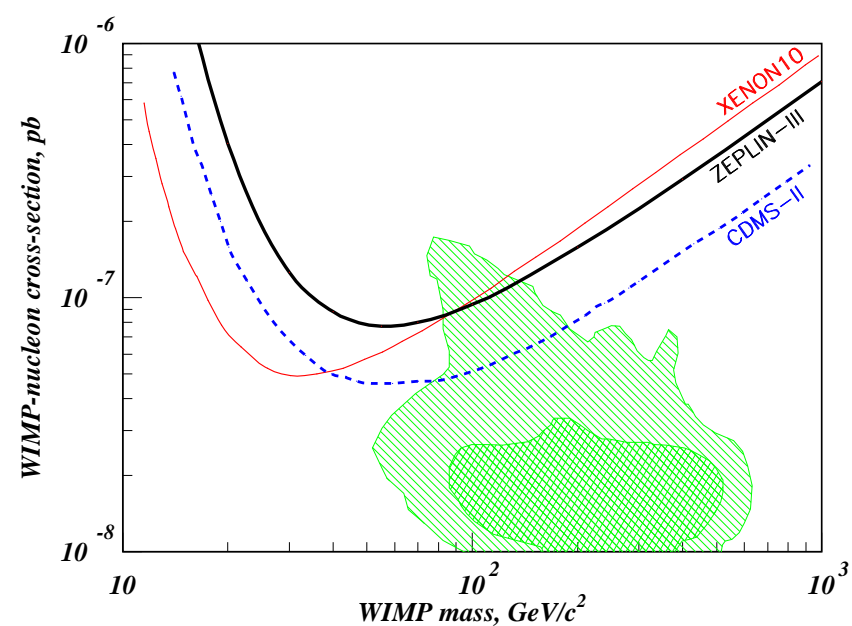

FIG. 17: 90\% confidence interval upper limit to the WIMPnucleon elastic scattering cross-section as derived from the first science run of ZEPLIN-III for a spin-independent interaction. For comparison, the experimental results from XENON10 [10, 27] and CDMS-II [28] are also shown. Note that the XENON10 curve is a 1-sided limit, corresponding approximately to an $85 \%$ confidence 2 -sided limit [10]. CDMS-II and our result are both $90 \% 2$-sided limits. The hatched areas show $68 \%$ and $95 \%$ confidence regions for the neutralinoproton scattering cross-section with flat priors as calculated in Constrained MSSM [30].

mismatch between the nuclear recoil spectrum shown in the AmBe calibration data and the Monte Carlo simulation. A careful and thorough analysis of efficiency factors and threshold effects (including the use of alternative data-sets with different thresholds, systematic changes to software cuts and thresholds, visual scanning and manual analysis of large samples of data and modelling and direct verification of the performance of the DAQ) did not resolve this mismatch. As a more credible alternative explanation the possibility of a non-linearity in the nuclear recoil energy scale has been studied. Non-linearity as such is not unexpected and, indeed it would be surprising if it did not exist at low energy, and a similar approach has been used by others for xenon [12]. Using this analysis it has been possible to reconcile the data with a non-linearity setting in at the same energy as in 12 but with a more significant effect at lower energies. In itself this may not be surprising given the very different operating conditions within ZEPLIN-III and XENON10: the most obvious being that the electric field in the liquid is 6 times stronger in the former. Indeed, there are other clear differences in the performances of the two instruments. However, it is clear that the physics underlying the low-energy performance is poorly understood. This is true of both the response to electron recoils [11] and to nuclear recoils [12. As a point of reference, if the mismatch between the AmBe simulation and the data were interpreted solely as an instrument efficiency, the effect on the upper limit would not have been dramatic $(<40 \%$ increase) as this approach has a better effective threshold for nuclear recoils but a poorer efficiency.

The analysis presented is not blind as one of the analysis routines was changed after opening of the full data-set as was the limit setting procedure. In applying the limit analysis no use was made of any background estimates (neither electron-recoil or neutron scattering) and this was done deliberately to avoid underestimating the upper limit.

\section{Acknowledgements}

The UK groups acknowledge the support of the Science \& Technology Facilities Council (STFC) for the ZEPLIN-III project and for maintenance and operation of the underground Palmer laboratory which is hosted by Cleveland Potash Ltd (CPL) at Boulby Mine near Whitby, on the North-East coast of England. The project would not be possible without the cooperation of the management and staff of CPL. We also acknowledge support from a Joint International Project award, held at ITEP and ICL, from the Russian Foundation of Basic Research (08-02-91851 KO a) and the Royal Society. We are indebted to our colleagues at ITEP, D.Yu Akimov, V. Belov, A. Burenkov and A. Kobyakin for their contributions. LIP-Coimbra acknowledges financial support from Fundação para a Ciência e Tecnologia (FCT) through the project-grants POCI/FP/81928/2007 and CERN/FP/83501/2008, the postdoctoral grant $\mathrm{SFRH} / \mathrm{BPD} / 27054 / 2006$, as well as the PhD grants SFRH/BD/12843/2003 and SFRH/BD/19036/2004. The University of Edinburgh is a charitable body, registered in Scotland, with the registration number SC005336.
[1] G.J. Alner et al., Astropart. Phys. 23, 444 (2005)

[2] G.J. Alner et al., Astropart. Phys. 28, 287 (2007)

[3] H.M. Araújo et al., Astropart. Phys. 26, 140 (2006)

[4] D.Yu. Akimov et al., Astropart. Phys. 27, 46 (2007)

[5] H.M. Araújo et al., Nucl. Instrum. Meths. A 521, 407 (2004)

[6] B.A. Dolgoshein, V.N. Lebedenko, B.U. Rodionov, JETP Lett.11 513 (1970)
[7] V.N. Solovov et al., In preparation (2009)

[8] A. Lindote et al., Nucl. Instrum. Meths. A 573, 200 (2007)

[9] F. Neves et al., arXiv:0905.2523 (2009)

[10] J. Angle et al., Phys. Rev. Lett. 100, 021303 (2008)

[11] T. Shutt et al., Nucl. Phys. B Proc. Suppl. 173, 160 (2007)

[12] P. Sorensen et al., Nucl. Instrum. Meths. A 601, 339 
(2009)

[13] http://wwwasdoc.web.cern.ch/wwwasdoc/hbook_html3 /hboomain.html

[14] http://wwwasd.web.cern.ch/wwwasd/paw/

[15] http://root.cern.ch/

[16] S. Agostinelli et al., Nucl. Instrum. Meths. A 506, 250 (2003)

[17] P.F. Smith, Astropart. Phys. 16, 75 (2001)

[18] Cross-Section Evaluation Working Group (http://www.nndc.bnl.gov/csewg/); $\quad$ EDFV/B-VI and EDFV/B-VI data files may be accessed at National Nuclear Data Center (http://www.nndc.bnl.gov)

[19] D. Akimov et al., Phys. Letts. B 524, 245 (2002)

[20] E. Aprile el al., Phys, Rev. D 72, 072006 (2005)

[21] V. Chepel el al., Astropart. Phys. 26, 58 (2006)
[22] B. Edwards et al., Astropart. Phys. 30, 54 (2008)

[23] G.J. Feldman, R.D. Cousins, Phys. Rev. D 57, 3873 (1998)

[24] J.D. Lewin, P.F. Smith, Astropart. Phys. 6, 87 (1996)

[25] R.H. Helm, Phys. Rev. 104, 1466 (1956)

[26] L. Roszkowski, R. Ruiz de Austri, and R. Trotta, J. High Energy Phys. 07, 075 (2007)

[27] E. Aprile el al., Phys. Rev. C 79, 045807 (2009)

[28] Z. Ahmed et al., Phys. Rev. Letts. 102, 011301 (2009)

[29] V.N. Lebedenko et al., arXiv:0901.4348. (2009)

[30] R. Trotta et al., J. High Energy Phys. 12, 24 (2008)

[31] This has been verified by subsequent direct analysis and details will be published elsewhere 